Original Research Paper

\title{
Lecturer Performance Analysis using Multiple Classifiers
}

\author{
Hawraz Ahmad and Tarik Rashid \\ Software Engineering, College of Engineering, Salahaddin University-Erbil, Hawler, Kurdistan Region, Iraq
}

Article history

Received: 12-05-2015

Revised: 11-06-2016

Accepted: 13-06-2016

Corresponding Author:

Tarik Rashid

Software Engineering, College

of Engineering, Salahaddin

University-Erbil, Hawler,

Kurdistan Region, Iraq

E-mail: tarik.rashid@su.edu.krd

\begin{abstract}
Lecturer performance analysis has enormous influence on the educational life of lecturers in universities. The existing system in universities in Kurdistan-Iraq is conducted conventionally, what is more, the evaluation process of performance analysis of lecturers is assessed by the managers at various branches at the university andin view of that, in some cases the outcomes of this process cause a low level of endorsement among staffs who believe that most of these cases are opinionated. This paper suggests a smart and an activesystem in which both unique and multiple soft computing classifier techniques are used to examine performance analysis of lecturers of college of engineering at Salahaddin University-Erbil (SUE). The dataset collected from the quality assurancedepartment at SUE. The dataset composes of three sub-datasets namely: Student Feedback (FB), Continuous Academic Development (CAD) and lecturer's portfolio (PRF). Each of the mentioned subdatasets is classified with a different classifier technique. FB uses BackPropagation Neural Network (BPNN), CAD uses Naïve Bayes Classifier (NBC) and the third sub-dataset uses Support Vector Machine (SVM) as a classifier technique. After implementing the system, the results of the above sub-datasets are collected and then fed as input data to BPNN technique to obtain the final result and accordingly, the lectures are awarded, warned or punished.
\end{abstract}

Keywords: Lecturer Performance, Soft Computing, Multiple Classifiers

\section{Introduction}

The development of lecturer performance analysis has excessive impact on universities, as it monitors the scholastic value through inspiring the awareness of researchers and academics and encouraging the value of education and training at universities. The assessment process of academics' level along with their teaching performance is considered to be a relatively restricted process inside an institution which conducts the assessment on its academics and researchers primarily to bolster and reassure that the main learning and teaching objectives of the institution are realized (Chaudhari et al., 2012).

The Quality Assurance Division (QAD) at the higher education and scientific research ministry in Kurdistan has the obligation to consider and certify teaching and research qualities of universities. The evaluation processcan be performed through holding a yearly basis competition of national ranking on academic and research activities and achievements of universities. Thus, there is an urgent need to redevelop the current system as it has major flaws and most notably, the evaluating process is operated by hand which is very prone to make mistakes and also it lacks to include intelligent and learning elements that can add generalization ability to the system during decision making process.

The two main contributions of this research work are as follows:

- To design, test and produce a consistent and useful system for evaluating academics' performance using an active and smart soft computing classifier technique

- To demonstrate that using a system that has multiple soft computing classifiers yields more reliable outcomes and precise accuracy than a system that doesn't have

In this research work, three significant measures (criteria) are used to assess the activities and performance of academics. Details of each key measure are included later in the paper. Also, unique and multiple classifiers to classify the performanceof lecturer are considered. The classifiers are BPNN, NBC and SVM.

This paper is structured as follows: In section 2, study background is presented, next, a brief background on each used technique is introduced, then, in section 4, 
the proposed technique and dataset are described, then after, in section 5 , the results are demonstrated, then followed by section 6 , which presents histogram and evaluation of results and finally, the main conclusions and future work points are outlined.

\section{Study Background}

It has been noticed from the collected research works in this study that few related research works were formerly piloted by means of subsystems methods to decide about the performance of lecturers at universities. Also the idea of using an intelligent system for assessing academics and their performance has not been realized in Kurdistan. Thus, it is crucial to use a system that has different subsets systems in this regard. Accordingly, the core of this work in this study attempts to normalize and use an appropriate system in order to solve the academic performance problem.

In the last several years, the applications of performance examination have attracted various researchers from different profession fields such as the application of learner performance (Amin and Khan, 2009; Abidin et al., 2008; Zarlis et al., 2011; Karamouzis and Vrettos, 2008; Do and Chen, 2013). This is reasonably similar to the application of academic performance (Chaudhari et al., 2012; Khan et al., 2011).

Fuzzy Expert System model has been proposed in (Chaudhari et al., 2012), to assess academic performance. The fields of the data sets covered feedback of learners, outcomes, attendance of learners, activities of teaching and learning process, development of academic teaching and activities. The data inputs are formed via fuzzification process into linguistic variables such as poor, average, good, very good and excellent. The crisp sets are transformed into the fuzzy set via using Trapezoidal function (type of membership function). Input and output membership functions are determined via fuzzy rules in which inference process is taken place (Chaudhari et al., 2012).

In addition, fuzzy logic technique is suggested as an expert system for assessing academic performance in (Khan et al., 2011). They utilized 99 propertieswhich were taken from a research work in (Amin and Khan, 2009). It was found that these properties greatly influenced academic performance. Membership functions and fuzzy sets are used via 'If else expert system approach' for mapping linguistic variables of academic performance and to make decisions.

Abidin et al. (2008), neural networks and Bayesian probabilities are proposed as a hybrid method for categorizing learner academic performance (Abidin et al., 2008). They implemented conventional neural network and a hybrid approach which involves Bayesian probability to initialize weights with back propagation feed forward neural network. They concluded that back propagation feed forward neural networks outperformed conventional neural networks.
Smooth Support Vector Machine as a data mining technique was presented for learner academic performance. Smooth Support Vector Machine is implemented for forecasting learner performance, whereas, kernel k-means approach is implemented for clustering similar features for a learner within a same cluster. The clustering approach is implemented over the data set. They used C4.5 decision tree approach to discover the logical rules of final grade of a learner. They selected Radial Basis Function to act as kernel function. They stated that Radial Basic Function can have few parameters and yield better results (Zarlis et al., 2011).

In the next section, the used soft computing techniques are introduced and described in details.

\section{Used Techniques}

This paper uses both unique and multiple classifier techniques. The used techniques are BPNN, NBC and SVM classifiers. These techniques are described briefly in the following sub sections.

\section{Backpropagation Neural Network}

BPNN is designed to overcome the inability of single layer perceptron. The structure of BPNN is the same as a human brain where there are interconnections. The structure of the network is consisted of three layers namely; input layer, hidden layer and output layer. The neurons in one layer are linked to neurons in the subsequent layer via connected weights. Each neuron has two jobs before it sends its output to other layer neuron. First, it is the multiplicative summation of the inputs and weights, then second, applying an activation function to the result (Ahmed, 2013). The network has training sessions where it trains itself then test samples are applied to it for checking the recognition accuracy.

\section{Naïve Bayes Classifier}

Naïve Bayes models are also called simple Bayes or independence Bayes. In machine learning, naïve Bayes classifiers are a family of straightforward probabilistic classifiers focused on applying Bayes' theorem on features independently. Naïve Bayes is regarded as the modest brand of Bayesian network in which all properties can beself-regulating and are straightforwardlyassociatedwith labels. This is known as conditional independence (Hand and Yu, 2001; Zhang, 2004; Rish, 2001).

Assuming the classification variable is signified by $\mathrm{C}$, given that the $\mathrm{C}$ value is denoted by $\mathrm{c}$ and supposing we have only two labels, namely; the positive label $(+)$ and the negative label(-). Let's also assume that attribute values $\left(x_{1}, x_{2}, \ldots x_{n}\right)$ can be denoted by an example $E$, where, $x_{i}$ represents the attribute value of $X_{i}$.

Based on the rule of Bayes, the probability of $E=\left(x_{1}\right.$, $\left.x_{2}, \ldots, x_{n}\right)$ included in labelc can be expressed via (1) (Hand and Yu, 2001; Zhang, 2004; Rish, 2001): 


$$
p(c \mid E)=\frac{p(E \mid c) p(c)}{p(E)}
$$

Note that $p(E \mid c)$ is the probability of a selected exampleE included in a label $c, p(E \mid c)$ is probability of generating a selected instance included in $E$, given the label $c, p(c)$ is probability of occurrence of the label $c$ and $p(E)$ is probability of a selected example included in $E$. note that the example $E$ is categorized as $C$ which is a positive label $(+)$ (Hand and Yu, 2001; Zhang, 2004; Rish, 2001):

$$
f b(E)=\frac{p(C=+\mid E)}{p(C=-\mid E)} \geq 1
$$

$f b(E)$ represents a Bayesian classifier. Suppose that all properties are self-determiningand assuming the class variable value can be expressed by:

$p(E \mid c)=p\left(x_{1}, x_{2}, \ldots, x n \mid c\right)=\prod_{i=1}^{n} p(x i \mid c)$

The resulting classifier equation can be expressed as follows (Hand and Yu, 2001; Zhang, 2004; Rish, 2001):

$$
f n b(E)=\frac{p(C=+)}{p(C=-)} \prod_{i=1}^{n} \frac{p(x i \mid C=+)}{p(x i \mid C=-)}
$$

The function $f n b(E)$ in Equation (4) represents a naïve Bayesian classifier. In order to get details about NBC, paper articles (Hand and Yu, 2001; Zhang, 2004; Rish, 2001) are recommended for reading.

\section{Support Vector Machine}

This is another type of machine learning technique which was coined by Vapnik (1999). SVM uses a vector as an input and predicts a class label for the presented inputs. The SVM technique can be used to tackle various tasks such regression, classification and recognition (Boser et al., 1992). The average error between input and their target vector is reduced, simply because the classification via SVM depends on the concept of Structural Risk Minimization (Huang et al., 2004).

In the SVM, hyperplanes are used for the input data which may be a line in two dimensions space or a plane in three dimensions space or a hyperplane in higher dimensions to separate classes by finding the maximummargin of a hyperplane.

SVM can be implemented via three phases, the intention of the first phase is to maximize the margin of thehyperplane. The second phase aims to plan the input space to feature space that can be separated by a straight line. The final phase attempts to use the 'kernel trick' to get results in the first and the second phases
(Valstar and Pantic, 2005). The most widely used kernel functions are (Hsu et al., 2010):

- Linear:

$K\left(x_{i}, x_{j}\right)=X_{i}^{T} X_{j}$

- Sigmoid:

$K\left(x_{i}, x_{j}\right)=\tanh \left(\gamma X_{i}^{T} X_{j}+r\right)$

- Polynomial:

$K\left(x_{i}, x_{j}\right)=\left(\gamma X_{i}^{T} X_{j}+r\right)^{d}, \gamma>0$

- Radial Basis Function:

$K\left(x_{i}, x_{j}\right)=\exp \left(-\gamma\left\|X_{i}-X_{j}\right\|^{2}\right), \gamma>0$

In this study, polynomial is used as a kernel function. The above mentioned techniques are used in this study for sub-datasets separately as it is presented in the next section. Papers (Hsu et al., 2010; Chang and Chih, 2013; Rashid and Hamid, 2014) are recommended for reading the SVM technique.

\section{Proposed Technique}

The QAD in the college of engineering at SUE provided data as spreadsheets and hard copies. Manual transfer preprocesses are done to be suitable for the system. The dataset composes of three sub-datasets as mentioned earlier called FB, PRF and CAD. Performances of lecturers are analyzed by the above three ways. Feedbacks of students are taken into consideration. Lecturer's portfolios are also one way to take the performance and quality of lecturers into consideration. The portfolio is a bundle of questions that a committee from each department in the college decides about the lecturer, then the portfolio form is handed over to the quality assurance of the college. CAD is the last way to observe the performance and quality of the lecturers in the college. It includes the participation of lecturers in the conferences and workshops as well as paper publications and holding seminars. Each subdataset is having its own features which are seen in the Table 1-3. FB consisted of 620 samples, each of which includes 12 features, while PRF and CAD are composed of 313 samples with 11 features and 342 sampleswith 3 features respectively. The sub-dataset samples are separated as $80 \%$ for training phase and $20 \%$ for testing phase. Each sub dataset has its own function of class labeling according to the policy of the quality assurance department at SUE. The scores of sub-datasets are 
collected and handed over to the college quality assurance department through which they are handed over to the university quality assurance department for the final score of the performance and quality of the lecturers. The class labeling functions of each subdataset is presented in the Table 4-6.

The final output and result depend on the results of the above three sub-datasets. The university has its own function to label the classes and depending on the labels that refer to performance of lecturers who may be awarded, warned, or punished so that to improve the quality and to make the lecturers be more serious in the teaching methodology and to make them be more satisfying. The labeling function is more described in the Table 7.

The proposed system is called a partitioned system since the dataset are not used all together, for each part there is a sub dataset that uses a specific technique. The system is best presented in Fig. 1 .
FB sub dataset uses BPNN classifier and its samples are divided between training set and testing set. The FB sub-system is then can be tested via the testing data and the results are stored to be used as one of those features of results sub dataset to get the final result. The same thing applied for the PRF sub dataset. It is partitioned into training and testing sets, the PRF sub system is using SVM technique as a classifier. The sub system is trained and eventually testing samples used to produce the result and then the result is stored to be used as a second feature in the results sub dataset to get the final result. The final sub dataset is $\mathrm{CAD}$ which uses $\mathrm{NBC}$ as a classifier technique. Again it is partitioned to perform both training and testing. Finally the results are then stored to be used as one of those features in results sub dataset to get the final output class label.

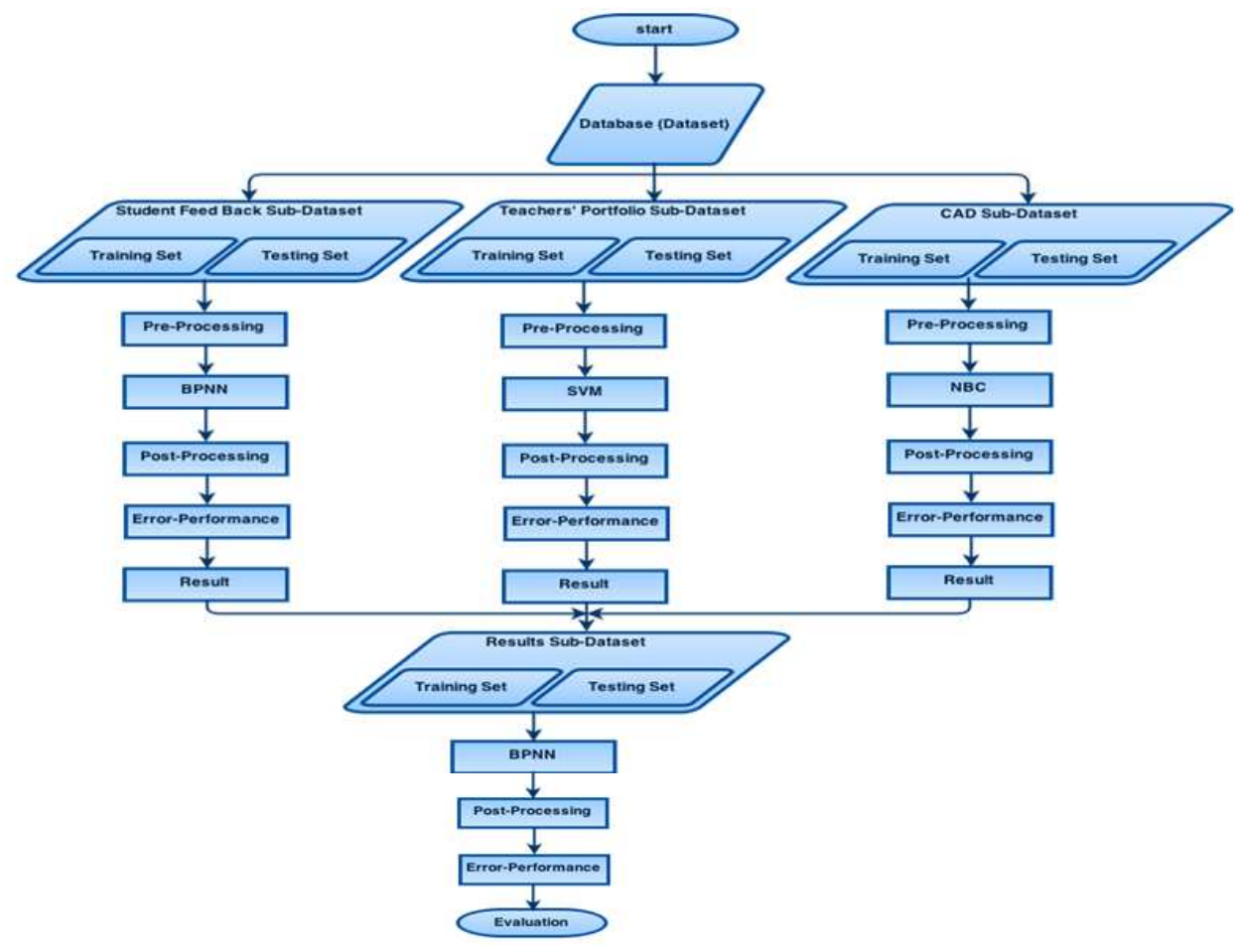

Fig. 1. Proposed system structure

Table 1. Shows student FB subdatasetproperties

\begin{tabular}{ll}
\hline Fields & Explanation \\
\hline FB-1 & Do you think that the course purpose and objective are clear? \\
FB-2 & Do you think that the subject contents were suitable? Do you think that the contents were relevant to the goals of the courses? \\
FB-3 & Do you think that lecturer has tried to prepare the course book? \\
FB-4 & Do you think that the lecturer put effort to explain basics and advanced topics throughout the duration of the course? \\
FB-5 & Do you think that the lecturer started and finished classes on time? \\
FB-6 & Do you think that the lecturer acted gently and dutifully all the way through classes? \\
FB-7 & Do you think that the used slides were flawless and attractive? \\
FB-8 & Do you think that the lecturer set aside a percentage of time for learners to ask questions and tried to respond learners' queries? \\
FB-9 & Do you think that the lecturer openly accepted the disparagement of learners? \\
FB-10 & Do you think that enough information was given about exams? \\
FB-11 & Do you think that the exam questions were related to the subject contents? \\
FB-12 & Do you think that the references were fresh and appropriate for the subject contents?
\end{tabular}


Table 2. Shows PRF sub dataset properties

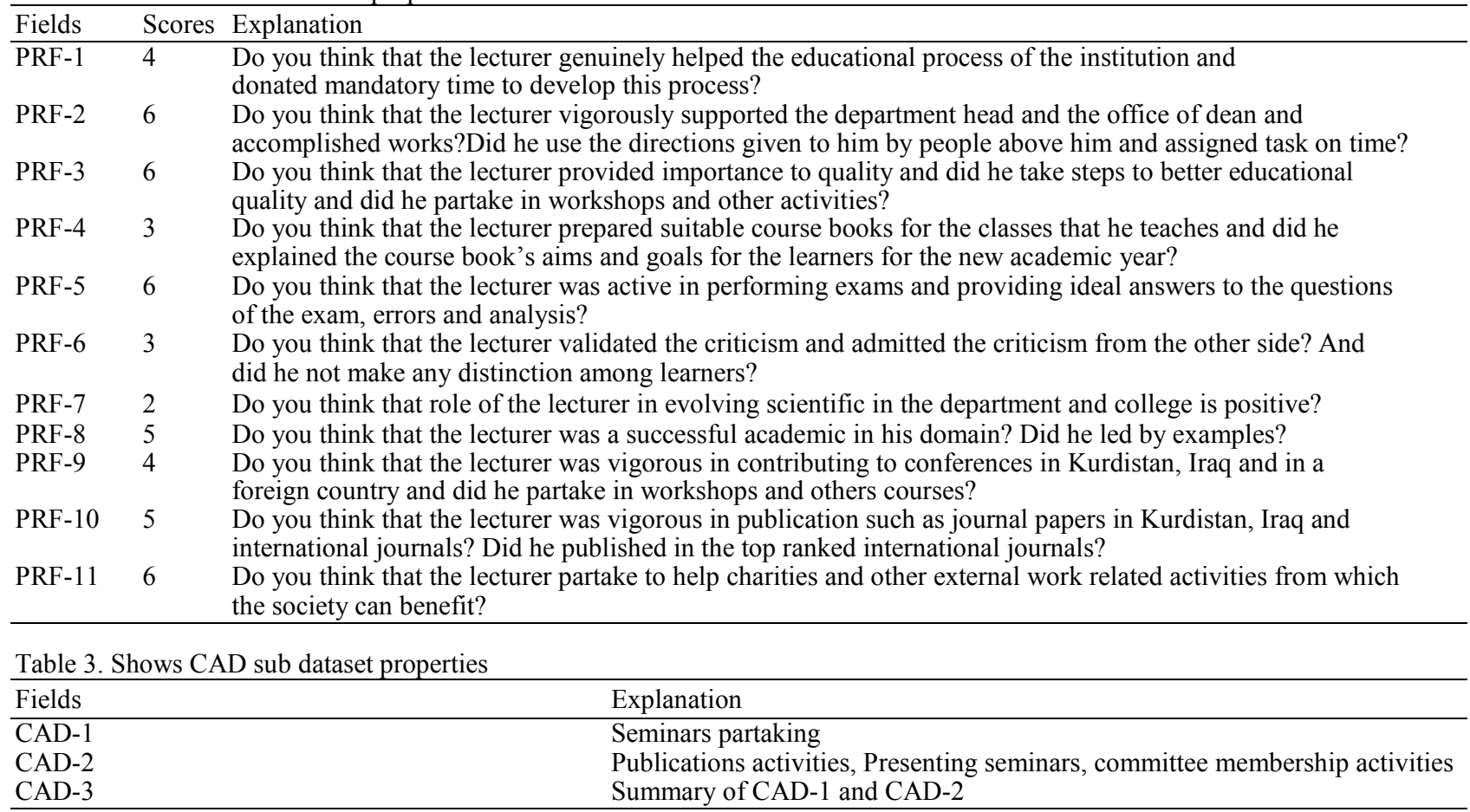

Table 4. Shows student FB class labeling

\begin{tabular}{llll}
\hline Class Label & Code & Scores & Explanation \\
\hline A $^{*}$ & AA & $>4.5$ & Very good \\
A & A & $>2.5$ & Good \\
B & B & $2-2.49$ & Medium \\
C & C & $1.5-1.99$ & Fair \\
D & D & $1-1.5$ & Late \\
\hline
\end{tabular}

Table 5. Shows PRF class labeling

\begin{tabular}{lll}
\hline Class Label & Scores & Explanation \\
\hline A & $4.5-5.0$ & Excellent \\
B & $4-4.4$ & Very good \\
C & $3-4$ & Good \\
D & $2-2.9$ & Medium \\
E & $1-1.9$ & Late \\
\hline
\end{tabular}

Table 6. Shows CAD class labeling

\begin{tabular}{lllllll}
\hline Class label & Code & Prof. & Assist. Prof. & Lec. & Assist. Lec. & Explanation \\
\hline A* & AA & $>80$ & $>70$ & $>55$ & $>45$ & Excellent \\
A & A & $60-79$ & $50-69$ & $35-54$ & $25-44$ & Very good \\
B & B & $45-59$ & $35-49$ & $24-34$ & $18-24$ & Good \\
C & C & $20-44$ & $20-34$ & $15-23$ & $12-17$ & Medium \\
D & D & $6-19$ & $6-19$ & $6-14$ & $6-11$ & Fair \\
E & E & $0-5$ & $0-5$ & $0-5$ & $0-5$ & Late \\
\hline
\end{tabular}

Table 7. Shows the final results of decision labeling functions

\begin{tabular}{lllll}
\hline CAD & FB & Portfolio & Class label & Explanation \\
\hline$A^{*}$ & $A^{*}$ & A & A & Thanks letter: From minister \\
$\mathrm{A}^{*}$ or $\mathrm{A}$ & $\mathrm{A}^{*}$ or $\mathrm{A}$ & $\mathrm{B}$ & $\mathrm{B}$ & Thanks letter: From the deanery office \\
$<=\mathrm{B}$ & $<=\mathrm{B}$ & $\mathrm{B}$ or C & $\mathrm{C}$ & Rights remain the same \\
$>=\mathrm{A}$ & $>=\mathrm{A}$ & $\mathrm{D}$ & $\mathrm{C}$ & Rights remain the same \\
$<=\mathrm{B}$ & $<=\mathrm{B}$ & $\mathrm{D}$ & $\mathrm{D}$ & Warning \\
$>=\mathrm{A}$ & $>=\mathrm{A}$ & $\mathrm{E}$ & $\mathrm{D}$ & Warning \\
$<=\mathrm{B}$ & $<=\mathrm{B}$ & $\mathrm{E}$ & $\mathrm{E}$ & Firm warning \\
\hline
\end{tabular}


The results of the sub systems will be collected and will be fed as a test set to the results sub system. The Results sub system uses BPNN as classifier technique. The dataset already has training set to train the sub system. After training, the collected results from the above FB, PRF and CAD subsystems will be used as test samples to get the final result.

\section{Results}

After applying the described techniques using both phases of training and testing inall subsystems, henceforward the outcomescan be shown in the following tables. The input features, classifier parameters, output class labels and output results in both phases are shown in this section. Each subsystem has two models differentiated by changes in the classifier parameters. Each subsystem has total of 313 instances which is partitioned into 250 instances for training phase (approximately $80 \%$ of total instances) and 63 instances for testing phase (approximately 20\% of total instances). More details on subsystems' results provided below.

\section{FB Subsystem Results}

In the previous section, it is mentioned that FB subsystem uses BPNN as a classifier. Two different models exist for this subsystem which they are differentiated by classifier parameters. Table 8 shows the classifier parameters of the first model which are the input features, output class, hidden layer, learning rate, momentum and epoch. After applying the parameters on both phases in the first model, the outcome results can be demonstrated in Table 9. The tables of both models show results ofboth phases: Time To Build Model (TTBM) and Root Mean Square Error (RMSE), the Mean Absolute Error (MAE), percentage of Correctly Classified Instances (CCI), percentage of Incorrectly Classified Instances (ICI). It is worth indicating that the first model uses one hidden layer composed of 8 neurons.

Next, in the second model, parameter values of the classifier will get changed to get better results. The classifier parameters and the resultsafter applying the parameters to the network are presented in Table 10 and 11 respectively.

It can be seen that the two models have the same testing result but when they are compared in terms of speediness, the second model which uses two hidden layers with 12 and 8 neurons gives the same result in a faster fashion.

\section{PRF Subsystem Results}

The PRF subsystem uses SVM as classifier technique. The PRF subsystem has two models which have different classifier parameter values. For both models, the input features, output class, degree of kernel type, kernel type, gamma, coefficient and cost explicitly are shown. Table 12 shows the classifier parameters of first model of PRF subsystem. After applying the parameters on both phases of the first model, the outcomes are shown in Table 13.

The second model for PRF subsystem has a different kernel type with different values of cost and coefficient. The classifier parameters are shown in Table 14. The result of the second model of both phases are shown in Table 15. Clearly, the second model is faster to get the same result as in the first model.

\section{CAD Subsystem Results}

The CAD subsystem uses CAD sub-dataset as input. The classifier technique for this subsystem is NBC. The system has only one model since there are not different options of NBC classifier parameters. The input features, output classand function parameters of the subsystem are presented in Table 16.

After applying the above parameters for both training and testing phases in this subsystem, the outputs for both phases are shown in Table 17. The CAD subsystem has the worst results as it is compared with the other subsystems mentioned in this study. The main reason behind this issue is due to input features which are totally of 3 features and these features are not capable enough to identify the classes.

\section{Results Subsystem}

The results of the sections A, B, C subsystems are then collected and fed as inputs to the test phase of the Result subsystem as it is shown in the structure of the proposed system. The features RFB, RCAD and RPRF are referred to FB subsystem result, $\mathrm{CAD}$ subsystem result and PRF subsystem result respectively. The Results subsystem uses the same classifier as FB subsystem which is BPNN. This subsystem uses two different models with different classifier parameters to obtain and choose the best and most accurate parameters. Table 18 shows only one hidden layer with 10 neurons. The result of both phases for the first model are presented in Table 19. The TTBM, CCI, ICI, MAE and RMSE values are presented in results table for both training and testing phases.

The second model of Results subsystem uses different classifier parameters which are presented in Table 20. The second model uses two hidden layers with 8 neurons for each layer and different classifier parameters from the first model. The result of the second model of Results subsystem is presented in Table 21 . 
Hawraz Ahmad and Tarik Rashid / Journal of Computer Sciences 2016, 12 (5): 255.264 DOI: 10.3844/jessp.2016.255.264

Table 8. First model FB subsystem-BPNN classifier parameters

\begin{tabular}{lll}
\hline Input features & Output (Class) & Hidden
\end{tabular}

FB1-FB12 AA, A, B, C, D

8

Learning rate

Momentum

Table 9. First model FB subsystem

\begin{tabular}{llllll}
\hline Phase & TTBM $(\mathrm{Sec})$ & CCI $(\%)$ & ICI (\%) & MAE & RMSE \\
\hline Training & 3.49 & 99.20 & 0.8000 & 0.0049 & 0.0556 \\
Testing & 3.89 & 92.06 & 7.9365 & 0.0360 & 0.1671 \\
\hline
\end{tabular}

Table 10. Second model FB subsystem-BPNN classifier parameters

\begin{tabular}{llllll}
\hline Input Features & Output (Class) & Hidden & Learning rate & Momentum & Epoch \\
\hline FB1-FB12 & AA, A, B, C, D & 12,8 & 0.6 & 0.3 & 500 \\
\hline Table 11. Second model Fb subsystem & & & & \\
\hline Phase & TTBM (Sec) & CCI (\%) & ICI (\%) & MAE & RMSE \\
\hline Training & 2.4 & 95.20 & 4.8000 & 0.0334 & 0.134 \\
Testing & 2.36 & 92.06 & 7.9365 & 0.0471 & 0.169 \\
\hline
\end{tabular}

Table 12. First modal PRF subsystem-SVM classifier parameters

\begin{tabular}{lllllll}
\hline Input Features & Output (Class) & Kernel type & Degree & Cost & Coef0 & Gamma \\
\hline PRF1-PRF11 & A, B, C, D, E & Linear & 3 & 0 & 0 & 0 \\
\hline
\end{tabular}

Table 13. First model PRF subsystem

\begin{tabular}{llllll}
\hline Phase & TTBM $(\mathrm{Sec})$ & CCI $(\%)$ & ICI (\%) & MAE & RMSE \\
\hline Training & 2.61 & 97.60 & 2.4000 & 0.0487 & 0.1104 \\
Testing & 2.02 & 98.41 & 1.5873 & 0.0626 & 0.1299 \\
\hline
\end{tabular}

Table 14. Second model PRF subsystem-SVM classifier parameters

\begin{tabular}{lllllll}
\hline Input features & Output (Class) & Kernel Type & Degree & Cost & Coef0 & Gamma \\
\hline PRF1-PRF11 & A, B, C, D, E & Polynomial & 3 & 1 & 5 & 0
\end{tabular}

Table 15. Second model PRF subsystem-results of training and testing phase

\begin{tabular}{llllll}
\hline Phase & TTBM $(\mathrm{Sec})$ & CCI $(\%)$ & ICI $(\%)$ & MAE & RMSE \\
\hline Training & 2.16 & 97.60 & 2.4000 & 0.0485 & 0.1105 \\
Testing & 1.97 & 98.41 & 1.5873 & 0.0605 & 0.1263 \\
\hline
\end{tabular}

Table 16. CAD Naïve Bayes classifier parameters

\begin{tabular}{lll}
\hline Input features & Output (Class) & Function \\
\hline CAD1, CAD2, CAD3 & AA, A, B, C, D, E & Naive Bayes \\
\hline
\end{tabular}

Table 17. Results of training and testing phase of CAD subsystem

\begin{tabular}{llllll}
\hline Phase & TTBM $(\mathrm{Sec})$ & CCI $(\%)$ & ICI $(\%)$ & MAE & RMSE \\
\hline Training & 0 & 72.80 & 27.2000 & 0.1027 & 0.2513 \\
Testing & 0 & 74.60 & 25.3968 & 0.0885 & 0.2288 \\
\hline
\end{tabular}

Table 18. First model results subsystem-BPNN classifier parameters

\begin{tabular}{llllll}
\hline Input features & Output Class & Hidden & Learning rate & Momentum & Epoch \\
\hline RPRF-RCAD-RFB & A, B, C, D, E & 10 & 0.5 & 0.8 & 500 \\
\hline
\end{tabular}

Table 19. First model results subsystem-results of training and testing phase

\begin{tabular}{llllll}
\hline Phase & TTBM $(\mathrm{Sec})$ & CCI $(\%)$ & ICI $(\%)$ & MAE & RMSE \\
\hline Training & 1.63 & 100.00 & 0.000 & 0.0022 & 0.0034 \\
Testing & 1.55 & 98.41 & 1.5873 & 0.0086 & 0.0792 \\
\hline
\end{tabular}

Table 20. Second model results subsystem-BPNN classifier parameters

\begin{tabular}{llllll}
\hline Input features & Output (Class) & Hidden & Learning Rate & Momentum & Epoch \\
\hline RPRF-RCAD-RFB & A, B, C, D, E & 8,8 & 0.4 & 0.8 & 1000 \\
\hline
\end{tabular}


Table 21. Second model results subsystem-results of training and testing phase

\begin{tabular}{llllll}
\hline Phase & TTBM $(\mathrm{Sec})$ & CCI $(\%)$ & ICI $(\%)$ & MAE & RMSE \\
\hline Training & 3.54 & 100 & 0 & 0.0021 & 0.0038 \\
Testing & 3.50 & 100 & 0 & 0.0042 & 0.0302 \\
\hline
\end{tabular}

Table 22. Percentage of classification accuracy using multiple classifiers

\begin{tabular}{|c|c|c|c|c|c|c|c|c|}
\hline \multirow[b]{2}{*}{ Model } & \multicolumn{2}{|c|}{ Feedback (BPNN) } & \multicolumn{2}{|c|}{ Portfolio (SVM) } & \multicolumn{2}{|c|}{ CAD (NBC) } & \multicolumn{2}{|c|}{ Results (BPNN) } \\
\hline & Training & Testing & Training & Testing & Training & Testing & Training & Testing \\
\hline First Model & 99.20 & 92.06 & 97.60 & 98.41 & 72.80 & 74.60 & 100 & 98.41 \\
\hline Second Model & 95.20 & 92.06 & 97.60 & 98.41 & NULL & NULL & 100 & 100.00 \\
\hline
\end{tabular}

Table 23. Percentage of classification accuracy using unified classifiers

\begin{tabular}{|c|c|c|c|c|c|c|}
\hline \multirow[b]{2}{*}{ Model } & \multicolumn{2}{|l|}{ BPNN } & \multicolumn{2}{|l|}{ SVM } & \multicolumn{2}{|l|}{ NBC } \\
\hline & Training & Testing & Training & Testing & Training & Testing \\
\hline Feedback & 95.20 & 92.06 & 88.80 & 85.71 & 89.60 & 82.54 \\
\hline Portfolio & 98.00 & 96.83 & 97.60 & 98.41 & 88.40 & 77.78 \\
\hline CAD & 68.80 & 73.01 & 73.21 & 73.01 & 72.80 & 74.60 \\
\hline Results & 100.00 & 100.00 & 100.00 & 98.41 & 96.00 & 95.24 \\
\hline
\end{tabular}

\section{Histogram and Evaluation of Results}

In this section, figures show the experimental results of the subsystems. The classification accuracy of each subsystem for both phases is presented. Figure 2, shows training classification accuracy of models of subsystems.

Figure 3, shows testing classification accuracy of models of subsystems.

Finally, it is time to evaluate the results. It is noted that adding an extra hidden layer for subsystems that use BPNNas shown in Table 22, increases accuracy of testing phase results. The main reason behind having better accuracy in the second models of subsystems in which BPNN is used, is due to the weights which make the system get more generalized. The performance of BPNN in FB and Results subsystem are satisfying, whilst the performance of NBC in CAD subsystem is notable. The bad performance of $\mathrm{NBC}$ in $\mathrm{CAD}$ is related to the few input featuresand they are not clearly dependent on the class. Eventually, if all the best cases of the subsystems are used, the accumulated error until the final result achieved is $34.9206 \%$ (FB error $7.9365+$ PRF error 1.5873+ CAD error 25.3968).

Table 23, shows the summarized results of various experiments using unique classifiers of NBC, BPNN and SVM, each of whichwith all different subsets of data (feedback, portfolio, cad and the Results).

It is clearly seen in the above table that BPNN produced the best results with feedback data, whereas the SVM and BPNN producepromising results with portfolio, however, NBC produced the best results with CAD. Comparing the results of Table 23 with Table 22, it can be noted that using the multiple classifiers approach is more reliable and it produces better results than using the unique classifiers approach.

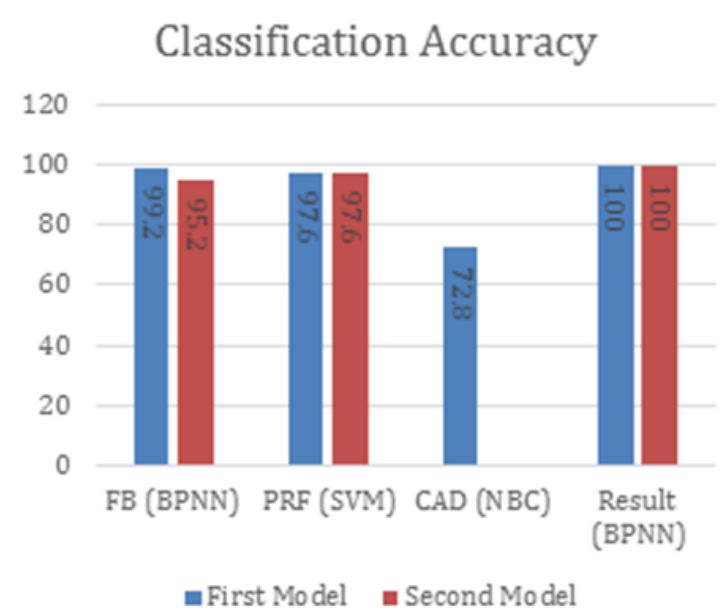

Fig. 2. Classification accuracy of training phase

\section{Classification Accuracy}

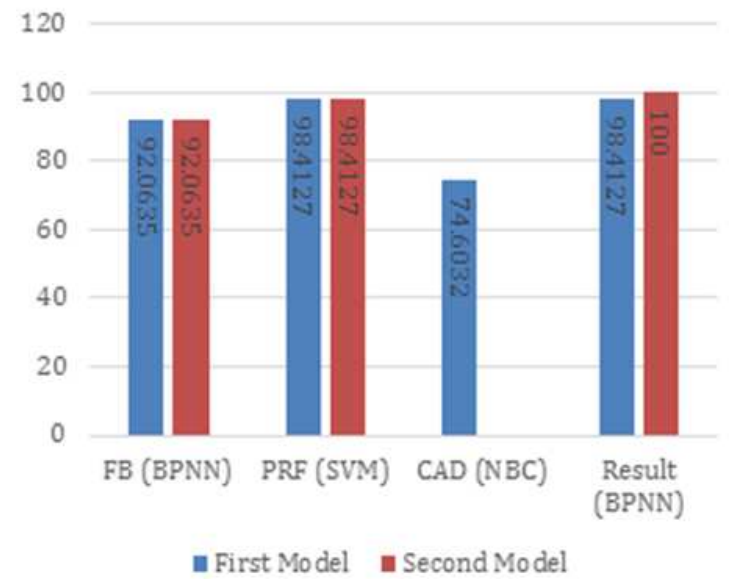

Fig. 3. Classification accuracy of testing 


\section{Conclusion and Recommendation}

During the course of our study in this area, it is found that there is little or no research works that were conducted previously using subsystems methods for evaluating the performance of lecturers and in the meantime, an automatic lecturer performance system has not been used in Kurdistan region in general or at Salahaddin University-Erbil in particular in particular, as a result, we consider it very important to use different subsets system for feature selection. This study challenges to device satisfactory system for assessing the performance of lecturers.

The output results from the system can make some clear points that can be analyzed easily. The following concluded points are taken from the results:

- The output class labeling functions play a great role in the performance and accuracy of the system. The nearness and the similarity between the class labeling functions hindered the system to get better accuracy

- Using the main dataset as separated sub-datasets causes the system to have error part in each subsystem and when they are all added they will cause accumulated error

- Adding an extra hidden layer to the subsystem using BPNN increases the accuracy of the system

- The relation of the features with the class label can influencethe systems as it is seen in CAD subsystem where they cannot purely recognize or detect the real class

- The subsystem that had badly affected the system is CAD where there is a few number of features that cannot fire the right class during training and testing phases

- Using themultiple soft computing classifier technique is more consistent and clear-cut in terms of precisionthan using the unique classifiers approach

There are some ways to get the system improved. The mainfuture recommendation points are outlined as follows:

- Alternate classifier algorithms can be used for the subsystems to improve the results especially for the CAD subsystem like tree classifiers, or other ANN classifiers, or fuzzy logic

- Combining the sub-datasets and using only one classifier for the system

- Increasing the samples by adding other college's data samples to make the system generalized

\section{Acknowledgement}

The authors of this paper would like to thank SUE and the Quality Assurance Division at SUE for their continuous effort on this research work.

\section{Author's Contributions}

Hawraz Ahmad: He collected, interpreted and analyzed the data. He implemented the design using Java programming language. He mainly wrote the first draft of the manuscript.

Tarik Rashid: He designed the main concepts and planned the steps of the proposed method. He also reviewed and analytically evaluated the manuscript for substantial scholarly content. He gave the final approval of the version for submission.

\section{Ethics}

There are no ethical issues that can be arisen after the publication of this work, as it isnovel and coversmaterials that are not published before. The corresponding author endorses that the other co-author has acknowledgedthe contents of the manuscript.

\section{References}

Abidin, A.I.Z., I.A.Setu, S.P. Yong, O.M. Foong and J. Ahmad, 2008. Classifying student academic performance: A hybrid approach. Proceeding of the International Multi Conference of Engineers and Computer Scientists, (CCS, 08), Hong Kong.

Ahmed, H.A., 2013. A proposed technique for human face behaviour recognition. College of Engineering. Salahaddin University, Erbil.

Amin, H.U. and A.R. Khan, 2009. Acquiring knowledge for evaluation of teachers' performance in higher education-using a questionnaire. Int. J. Comput. Sci. Inform. Security.

Boser, B.E., I.M. Guyon and V.N. Vapnik, 1992. A training algorithm for optimal margin classifiers. Proceedings of the 5th Annual Workshop on Computational Learning Theory, Jul. 27-29, New York, pp: 144-152. DOI: 10.1145/130385.130401

Chang, C.C. and J.L. Chih, 2013. LIBSVM-a library for support vector machines. Department of Computer Science, National Taiwan University, Taipei, Taiwan.

Chaudhari, O.K., P.G. Khot and K.C. Deshmukh, 2012. Soft computing model for academic performance of teachers using fuzzy logic. Brit. J. Applied Sci. Technol., 2: 213-226.

DOI: 10.9734/BJAST/2012/1237

Do, Q.H. and J.F. Chen, 2013. A neuro-fuzzy approach in the classification of students' academic performance. Comput. Intelli. Neurosci. DOI: $10.1155 / 2013 / 179097$ 
Hand, D.J. and K. Yu, 2001. Idiot's Bayes-not so stupid after all? Int. Stat. Rev., 69: 385-398. DOI: $10.1111 / \mathrm{j} .1751-5823.2001 . t b 00465 . x$

Hsu, C.W., C.C. Chang and C.J.Lin, 2010. A practical guide to support vector classification. Department of Computer Science, National Taiwan University, Taipei, Taiwan.

Huang, Z., H. Chen, C.J. Hsu, W.H. Chen and S. Wu, 2004. Credit rating analysis with support vector machines and neural networks: A market comparative study. Decision Support Syst., 37: 543-558. DOI: 10.1016/S0167-9236(03)00086-1

Karamouzis, S.T. and A. Vrettos, 2008. An artificial neural network for predicting student graduation outcomes. Proceedings of the World Congress on Engineering and Computer Science, Oct. 22-24, San Francisco, pp: 1-4.

Khan, A.R., H.U. Amin and Z.U. Rehman, 2011. Application of expert system with fuzzy logic in teachers" performance evaluation. Int. J. Adv. Comput. Sci. Applic., 2: 51-57.

Rashid, T. and S.A. Hamid, 2014. Support vector machines for predicting electrical faults. Int. J., 32: 1931-1941.
Rish, I., 2001. An empirical study of the naive Bayes classifier. T.J. Watson Research Center.

Valstar, M. and M. Pantic, 2005. Facial action unit detection using probabilistic actively learned support vector machines on tracked facial point data. Proceedings of the IEEE Computer Society Conference on Computer Vision and Pattern Recognition Workshops, Jun. 25-25, IEEE Xpress Press, San Diego, pp: 76-76. DOI: 10.1109/CVPR.2005.457

Vapnik, V., 1999. The Nature of Statistical Learning Theory. 2nd Edn., Springer Science and Business Media, New York, ISBN-10: 0387987800, pp: 314.

Zarlis, M., D. Hartama and R.S.E. Wani, 2011. Prediction of student academic performance by an application of data mining techniques. Proceedings of the International Conference on Management and Artificial Intelligence, (MAI, 11), IACSIT Press, Bali.

Zhang, H., 2004. The optimality of naive bayes. Am. Associat. Artifi. Intelli. 